\title{
A Proposed Smart E-Learning System Using Cloud Computing Services: PAAS, IAAS and Web 3.0
}

\author{
http://dx.doi.org/10.3991/ijet.v7i3.2066 \\ Mona Nasr and Shimaa Ouf \\ Helwan University, Helwan, Egypt
}

\begin{abstract}
E-learning systems need to improve its infrastructure, which can devote the required computation and storage resources for e-learning systems. Microsoft cloud computing technologies although in their early stages have managed to change the way applications are going to be developed and accessed. The objective of the paper is to combine various technologies to design architecture which describe E-learning systems. Web 3.0 uses widget aggregation, intelligent retrieval, user interest modeling and semantic annotation. These technologies are aimed at running applications as services over the internet on a flexible infrastructure. Cloud computing provides a low cost solution to academic institutions for their researchers, faculty and learners. In this paper we integrate cloud computing as a platform with web 3.0 for building intelligent e-learning systems.
\end{abstract}

Index Terms - web 3.0, e-learning, semantic annotation, intelligent retrieval, cloud computing, Microsoft windows azure, software as a services (SaaS), infrastructure as a services (IaaS), platform as a services (PaaS).

\section{I.INTRODUCTION}

With the rapid development of Internet, more and more people obtain useful information via the Internet [21]. The internet is an open system; a huge number of information is produced and updated onto it every day. [16] Elearning systems can be very beneficial to the learner if used properly. With the development of information society and acceleration of the pace of life, Learners are looking forward to have a learning schedule and network learning environment, which can meet their own needs. During the era of Web 2.0, Internet brought convenience to instructors and administrators of E-learning platforms. E-learning platforms mainly used interactivity of information. However learners still need a more intelligent efficient and personalized system [14]. Also, there is no Elearning, but has faced challenges in [5] optimizing resource allocations[3], dealing with dynamic demands on getting information and knowledge anywhere and anytime, handling rapid storage growth requirements, [14] cost controlling and greater flexibility, [9] improving its infrastructure[12], which can devote the required computation and storage resources Deficiency in learning content (The information may not inspire the users successfully),[8] Lack of personalization (The currently available platforms to learners are of the same content, so when learners have different learning demands and different learning roles, they have to enter different learning systems which reduce the learning desire and initiative), [7] Narrowness (E-learning systems are applied by small and specific groups). An integration between cloud computing and Web 3.0 meets this need of learners and proposes new concepts of information access models. The latest

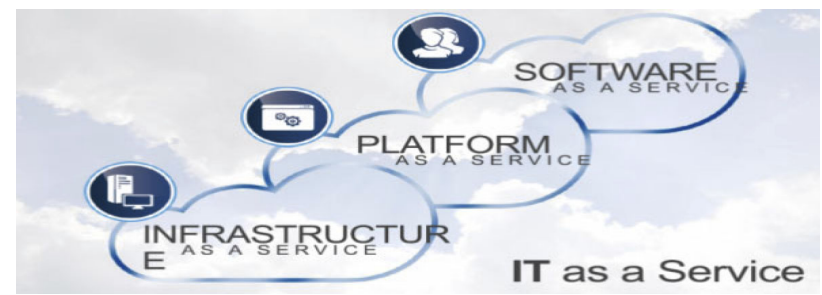

Figure 1. Cloud Computing Layers [21]

emerging area is cloud computing and Semantic Web. The languages that power the Semantic Web are RDF Resource Description Framework and OWL Web Ontology Language which helps in embedding Semantics in existing Web [13].

\section{II.CLOUD COMPUTING TODAY AND TOMORROW}

Cloud computing is an excellent alternative for academic institutes which are especially under budget shortage in order to build[1], host and operate their e-learning systems[6] effectively without spending [8] any more capital for the computers [1] purchase software, licensing new software, power, cooling[6], servers, storages, network devices, [18] technical team, maintenance team or do not have the resources and infrastructure needed to [15] run e-learning effectively[10]. If we look closely at the cloud, we will see three Cloud Computing services into three distinct categories [2] (Figure 1).

\section{A) Software as a Service (SaaS)}

The NIST definition of cloud computing defines: It is one of the clouds computing delivery model in which the consumer uses an application, but does not control the operating system, hardware or network infrastructure on which it's running. [17] It delivers applications to the browser of user or customer from the cloud. It helps academic institutes with limited IT resources to deploy and maintain needed software in a timely manner while, at the same time, reducing energy consumption and expenses. [20]

\section{B) Platform as a Service (Paas)}

The NIST definition of cloud computing defines Platform as a Service (SaaS): It is one of the cloud computing delivery model in which the consumer uses a hosting environment for their applications. [17] Platform as a Service (PaaS) deliver a computing platform and/or solution stack as a service, often consuming cloud infrastructure and sustaining cloud applications. It facilitates a reliable, secure and scalable development and deployment of applications without the cost and complexity of buying and 
PAPER

A Proposed Smart E-Learning System Using Cloud Computing Services: PAAS, IAAS ANd Web 3.0

managing the underlying infrastructure (hardware (server, storage and network), and associated software (operating systems virtualization technology, file system)).cloud platform provide all of the facilities required to support the complete life cycle [18] of building and delivering web applications and services entirely available from the Internet. [17]

\section{C) Infrastructure as a Service (Iaas)}

The NIST definition of cloud computing defines infrastructure as a Service (IaaS): It is one of the cloud computing delivery model in which the consumer uses "fundamental computing resources" such as processing power, storage, load balancers, a firewall, cables networking components or middleware. The consumer can control the operating system, storage, deployed applications and possibly networking components such as firewalls and load balancers, but not the cloud infrastructure beneath them. [22]

E-learning can use benefit from cloud computing using:

- Infrastructure: use e-learning on the provider's infrastructure.

- Platform: use and develop an e-learning based on the provider's development interface.

- Services: use the e-learning given by the provider.

\section{III.PROPOSED MODEL}

There have been many new advances in the computing field in recent times. Cloud Computing and Web 3.0 are two such areas that are beginning to significantly impact how we develop, deploy and use e-Learning application. Web 3.0 combines semantic Web with Web 2.0's tagging culture. It will use internet to make connections with information. Cloud Computing presents a new way of deploying applications. Today we can get Infrastructure as a Service (IaaS), Platform as a Service (PaaS) or Software as a Service (SaaS). There are elastic clouds where memory and processing power get allocated based on computing resources required at the time. Moreover, learning environment must be productive, scalable, flexible and adaptable towards learners' needs and learner preferred information and communication technologies. This raises the question of whether cloud computing and web3.0 can meet the indicated requirements. To answer this question, Figure 2 introduces the proposed model with new technologies, integration between cloud computing and web 3.0. In this paper the intelligent e-learning can use benefit from Microsoft windows azure using:

- Platform: use and develop intelligent e-learning based on the Microsoft windows azure development interface.

- Infrastructure: use intelligent e-learning on the Microsoft data center.

- Services: use intelligent e-learning given by the provider.

Firstly: when we use cloud computing as platform intelligent e-learning will be benefited from Microsoft windows azure as follows:

\section{A. Components of the Proposed Model:}

\section{1)The Web-based Services}

The model in Figure 2 provides learner with two kinds of contents, Learning content and Assessment content. Each content has different types of services such as:
- Learning services: provide registration, online course, interactive tutorial, course documents (is a repository for files that the educator have made available to the learner as a part of his/her course), announcements (displays information to the learner that the educators of the course want him/her to know), links (displays a list of useful URL links that have been identified by the course educators), learner papers (learners can post/upload requests files to the educator), and Semantic search (helps the learner to search for resources).

- Assessment services: provide exercises and questions for evaluation of the learner knowledge. During the learning process, a dynamic selection presentation of both contents will be accomplished. On other hand, our intelligent e-learning allows educators to create his/her course websites through a browser, and monitoring the learner performance. They have many services and tools such as: publish documents in any format (Word, PDF, Video,) to the learners, manage a list of useful links, compose exercises/questions, make announcements, and have learners submit papers.

\section{2)The Ontology based Model}

Before describing ontology based model, we will discuss learning environments illustrated in Figure 2. Course sequencing generally starts with the learner entity component that receives the learning contents, while the learner's behavior is being observed. The educator sends queries to the learning resources to search for learning content that is appropriate for the learner entity component. The ontological knowledge is added to the learning resources as a resource for contextual learning, and it may be searched by means of queries. The learner's performance is measured by the evaluation component, and the result is stored in the learner records database. The data in the database can be used by the educator component to locate a new content. Searching learning resources and sequencing a course can be done using a knowledge base of learning resources and a delivery component. To implement the knowledge base, first of all, the leaning resources have to be described by means of metadata. The metadata consists of the contextual knowledge of the learning resources, i.e., ontology in the model.[11]

\section{3)Cloud computing (Microsoft Windows Azure plat-} form)

Cloud computing as a platform is a new era of cloud computing offers the educational institutes a familiar development experience, on demand scalability, scale up when it need capacity and pull it back when it don't, cost savings, and reduced time to market for the intelligent eLearning application, all in a secure environment. It is a

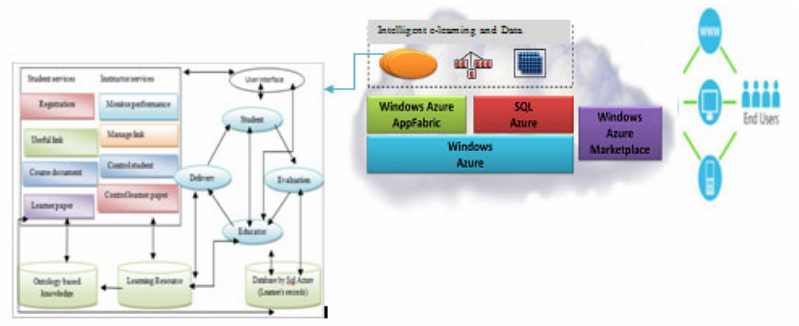

Figure 2. The Windows Azure platform supports intelligent eLearning application, data, and infrastructure in the cloud, together with a cloud marketplace. 
new instrument to host and deploy intelligent e-Learning application in more effective way. The Windows Azure platform today has four parts (Windows Azure, SQL Azure, Windows Azure AppFabric, Windows Azure Marketplace) [4] it is a windows environment for running intelligent e-Learning application and storing data on computers in Microsoft data centers.

\section{IV.WINDOWS AZURE}

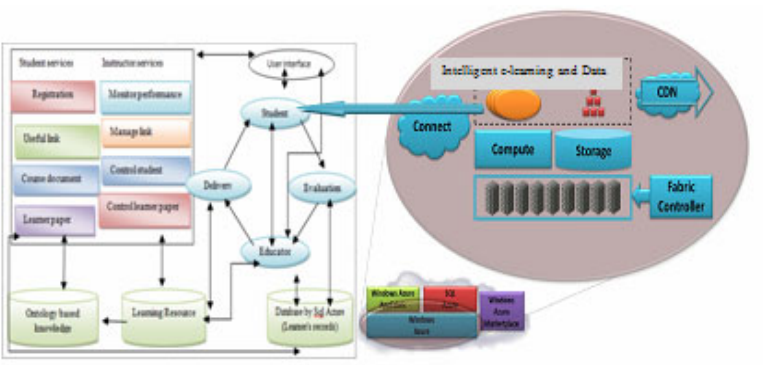

Figure 3. Windows Azure provides compute and storage services for intelligent e-learning in the cloud.

\section{A) Parts of Windows Azure today are as follows}

\section{1)Compute}

Intelligent e-learning built on the Windows Azure compute service is structured as more roles. When it executes, intelligent e-learning typically runs two instances of each role, with each instance running as its own virtual machine (VM). Figure 4 shows how this looks. Intelligent e-learning can be created using web roles: Web roles, intended primarily for running intelligent e-Learning. Each Web role instance has Internet Information Services (IIS) preconfigured to run inside it.

\section{2)Storage}

This service allows storing binary large objects (blobs), provides queues for communication between components of intelligent e-Learning application, and even offers a form of tables with a simple query language. Intelligent e-learning can access the Windows Azure storage service, and that can be done in the same way: using a RESTful approach (Figure 5).

\section{3)Fabric controller}

As the above figure suggests Windows Azure intelligent e-learning runs on a large number of machines. The fabric controller's job is to knit the machines in a single Windows Azure data center into a cohesive whole. Intelligent e-learning compute and storage services are then built on top of this pool of processing power (Figure 6).

\section{4)Content delivery network (CDN)}

Caching frequently accessed data closer to its users (learners and educators) speeds up access to that data. The Windows Azure CDN can do this for blobs, maintaining cached copies at sites around the world (Figure 7).

\section{5)Connect}

Windows Azure Connect make the connection between on-premises application and off-premises application easier. So, it's often useful for other academic institutes that are still on premises to interact with intelligent e-Learning based on cloud computing (Figure 8).

Running intelligent e-learning and storing data in the cloud can have clear benefits. Rather than buying, installing, and operating its own systems, for example, aca- demic institutes can rely on a cloud provider to do this for them. Also, academic institutes pay just for the computing and storage they use, rather than maintaining a large set of servers only for peak loads. And Windows Azure intelligent e-learning can scale better, be more reliable, and require less administration than academic institutes written using the traditional Windows Server programming model.

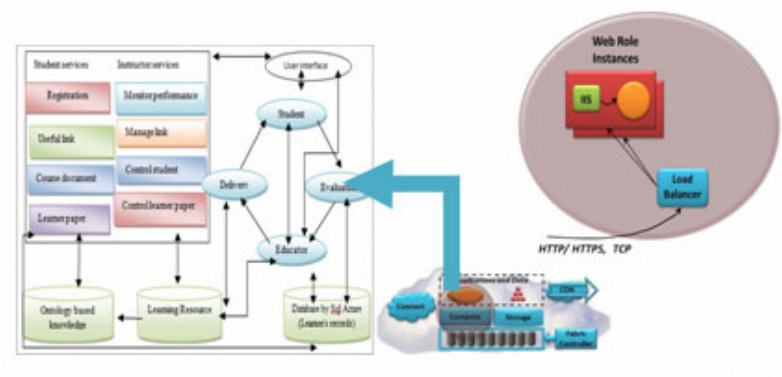

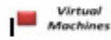

Figure 4. A running intelligent e-learning consists of Web role instances.

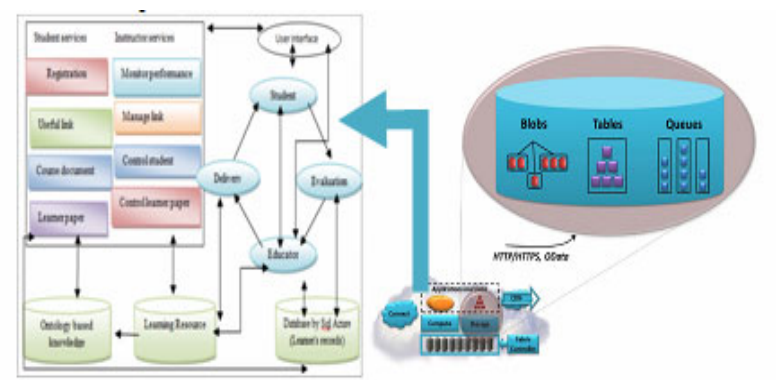

Figure 5. Windows Azure provides blobs, tables, and queues, all accessed in a RESTful style via HTTP, HTTPS, or OData.

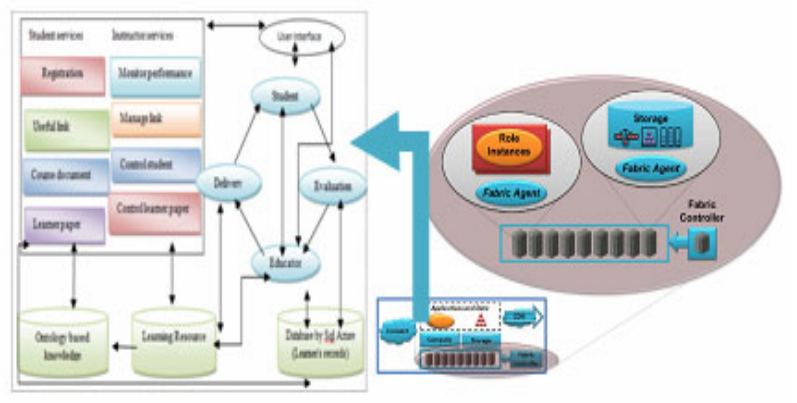

Figure 6. The fabric controller interacts with intelligent e-learning via a fabric agent.

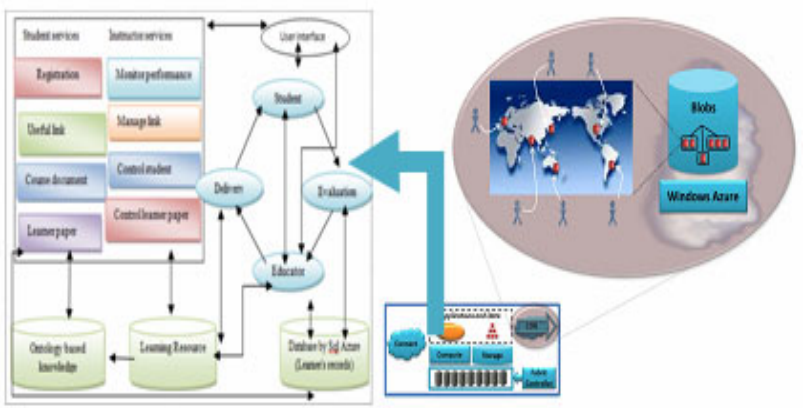

Figure 7. The Windows Azure CDN caches copies of blobs around the world, letting users access that information more quickly 


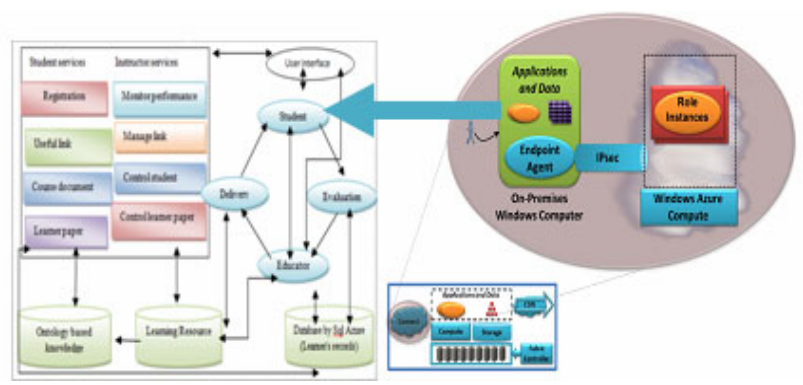

Figure 8. Windows Azure Connect allows IP level communication between on premises Windows machine and intelligent eLearning application using web 3.0

\section{A) SQL Azure}

Relational data services in the cloud based on SQL Server technologies. It is a highly available and scalable cloud database service built on SQL Server technologies. With SQL Azure, no need to install, setup, and patch or manage any software (Figure 9).

Figure 9.

\section{B) The components of SQL Azure today are as follows}

- SQL Azure Database provides a cloud based database management system (DBMS). This technology lets cloud intelligent e-learning store relational data on Microsoft servers in Microsoft data centers. As with other cloud technologies, academic institutes pay only for what it uses, increasing and decreasing usage (and cost) as the academic institutes' needs change. Using a cloud database also allows converting what would be capital expenses, such as investments in disks and DBMS software, into operating expenses.

- SQL Azure Reporting is a version of SQL Server Reporting Services (SSRS) that runs in the cloud. Intended primarily for use with SQL Azure Database, it allows creating and publishing standard SSRS reports on cloud data.

- SQL Azure Data Sync allows synchronizing data between SQL Azure Database and on premises SQL Server databases. It can also be used to synchronize data across different SQL Azure databases in different Microsoft data centers.

- SQL Azure is built on Microsoft SQL Server. As with SQL Server, we can create indexes and views, use stored procedures, define triggers, and more. Windows azure intelligent e-learning can access SQL Azure data using Entity Framework, ADO.NET, and other Windows data access interfaces.

\section{C) Windows Azure AppFabric}

Running intelligent e-learning and storing data in the cloud are both important aspects of cloud computing. It's also possible to provide cloud based infrastructure services. Filling this gap is the goal of Windows Azure AppFabric (Figure 10).

As the figure suggests, all of the components of Windows Azure AppFabric are built on Windows Azure. Those components are as follows:

\section{1)Service Bus}

Exposing intelligent e-Learning services on the Internet is harder than it might seem. The goal of Service Bus is to make this simpler by letting an intelligent e-learning

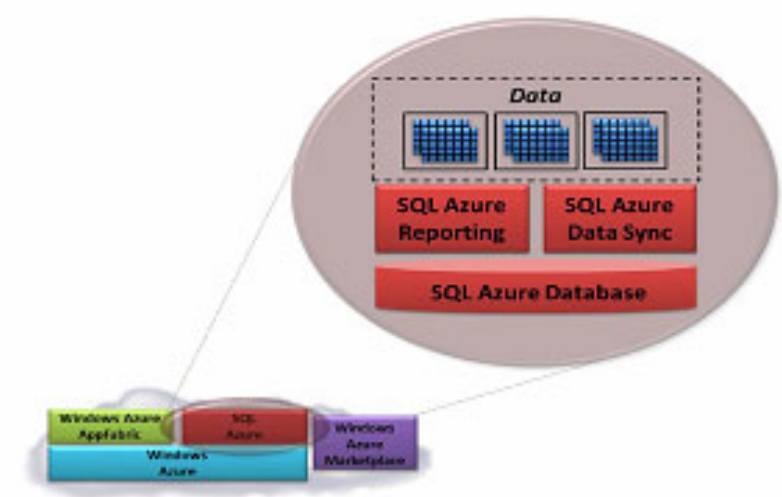

Figure 10. SQL Azure provides relational database services in the cloud.

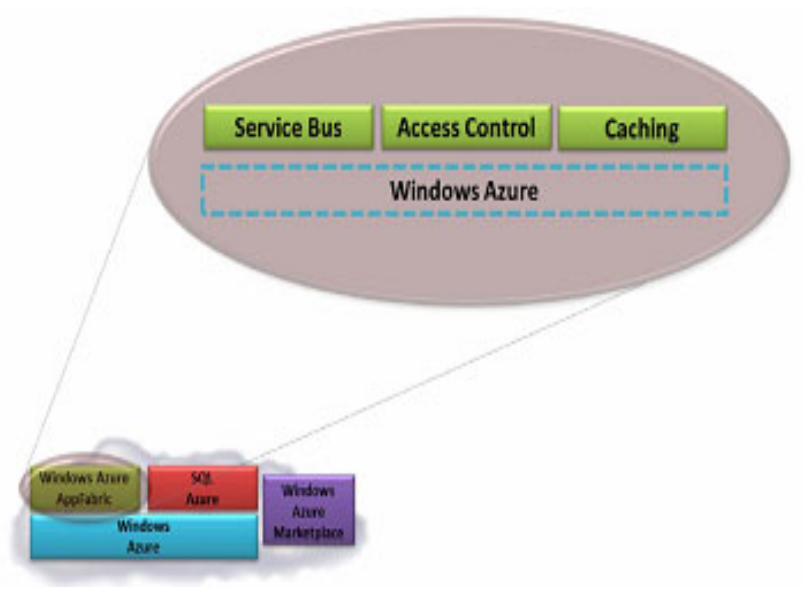

Figure 11. Windows Azure AppFabric provides Windows Azure based infrastructure services

expose endpoints in the cloud that can be accessed by other applications, whether on-premises or in the cloud. Each exposed endpoint is assigned a URL, which clients can use to locate and access the service. Service Bus also handles the challenges of dealing with network address translation and getting through firewalls without opening new ports for exposed applications.

\section{2)Access Control}

There are many ways for a user to get a digital identity today. The options include Active Directory, Windows Live ID, Google Accounts, Facebook, and more. If an intelligent e-learning lets users log in with any of these, the application's creator faces the daunting challenge of supporting this plethora of approaches. Access Control simplifies this by providing built in support for all of them. It also provides a single place for defining rules to control what each user is allowed to access.

\section{3)Caching}

It's common for intelligent e-learning to access the same data over and over. One way to speed up intelligent e-learning is to cache frequently accessed information, reducing the number of times that intelligent e-learning must query a database. The Caching service provides this and the performance boost it brings for Windows Azure intelligent e-Learning application using web 3.0. 
Secondly: when we use cloud computing as infrastructure, Servers are better (cheaper, faster, more fault tolerant, and more secure than traditional servers).

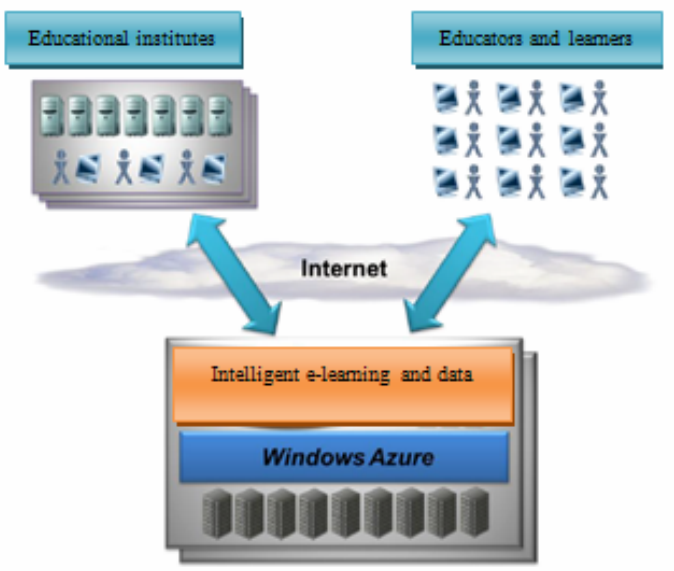

Figure 12. Intelligent e-learning application run in Microsoft data centers and are accessed via the Internet.

Thirdly: when we use cloud computing as services, we will use Microsoft Azure Market: educational institutes using the cloud to find, evaluates, and purchase our intelligent e-Learning application. The goal of Windows Azure Marketplace is to make these things easier to do (Figure 13).

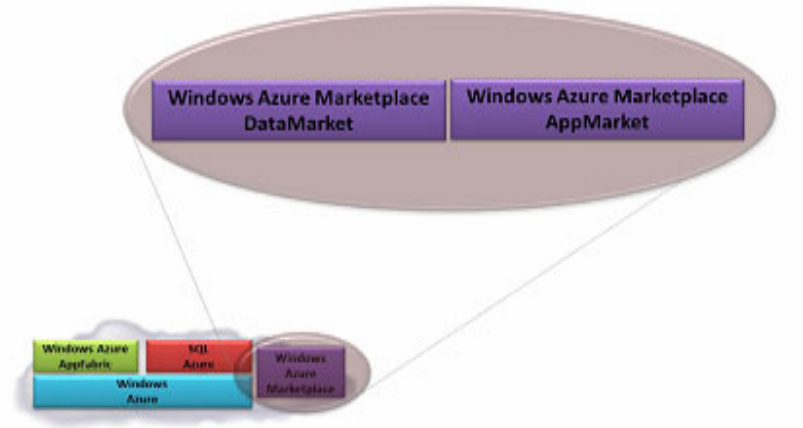

Figure 13. Windows Azure Marketplace provides an online marketplace for cloud intelligent e-Learning application and data.

D) The two parts of Windows Azure Marketplace are:

- DataMarket (originally codenamed “Dallas”), providing a way for content providers to make datasets available. Educational institutes can browse the offerings, and then purchase whatever they find useful.

- AppMarket, providing a way for creators of cloud intelligent e-Learning application to expose this application to potential educational institutes. AppMarket will be available sometime after DataMarket.

\section{V.CONCLUSION AND FUTURE WORK}

The development of e-learning cannot ignore the cloud computing and web3.0 trends. There are many benefits from using the integration between cloud computing and web3.0 for e-learning. Using cloud computing and web3.0 for e-learning influences the way an e-learning software projects are managed. There are specific tasks that deal with finding providers for cloud computing, depending on the requirements (infrastructure, platform or services). Also, the cost and risk management influences the way the e-learning based on integrating the cloud computing and web3.0 are managed. An intelligent elearning system based on an integration between cloud computing and web 3.0 has been developed in order to enhance the efficiency of learning environment, provide flourish; growing, up-to-date, self-regulated, stability, QoS-guaranteed (Quality of Service), equilibrium, reliability, scalability, time reduction, efficient resource use, flexibility, and sustainability of e- learning system. In the future we wish to develop an e-learning by using an integration between web 4.0 and cloud computing.

\section{REFERENCES}

[1] Al-Zoube, M. (2009). E-Learning on the Cloud. International Arab Journal of e-technology, 1(2), 58-64.

[2] Behrend, T. S., Wiebe, E. N., London, J. E., \& Johnson, E. C.(2011). Cloud computing adoption and usage in community colleges. Behaviour \& Information Technology, 30(2), 231-240. http://dx.doi.org/10.1080/0144929X.2010.489118

[3] Berl, A., Gelenbe, E., Di Girolamo, M., Giuliani, G., De Meer, H., Dang, M. Q., \& Pentikousis, K. (2010). Energy-efficient cloud computing. The Computer Journal, 53(7), 1045-1051. http://dx.doi.org/10.1093/comjnl/bxp080

[4] Chappell, D. (2010 Introducing the windows azure platform. Microsoft Corporation.

[5] Chang, V., \& Guetl, C. (2010). Generation y learning in the $21^{\text {st }}$ century: Integration of virtual worlds and cloud computing services. Proceedings of global learn asia pacific, 1888-1897.

[6] Chine, K. (2010). Learning Math and Statistics on the Cloud, Towards an EC2-Based Google Docs-like Portal for Teaching/Learning Collaboratively with $\mathrm{R}$ and Scilab.

[7] Choudhury, T., Consolvo, S., Harrison, B., Hightower, J., LaMarca, A., LeGrand, L., Hemingway, B. (2008). The mobile sensing platform: An embedded activity recognition system. Pervasive Computing, IEEE, 7(2), 32-41. http://dx.doi.org/10.1109/MPRV.2008.39

[8] Doelitzscher, F., Sulistio, A., Reich, C., Kuijs, H., \& Wolf, D. (2011). Private cloud for collaboration and e-Learning services: from IaaS to SaaS. Computing, 1-20.

[9] Ebner, M. (2009). Introducing live microblogging: how single presentations can be enhanced by the mass. Journal of research in innovative teaching, 2(1), 91-100.

[10] Ercan, T. (2010). Effective use of cloud computing in educational institutions. Procedia-Social and Behavioral Sciences, 2(2), 938942. http://dx.doi.org/10.1016/j.sbspro.2010.03.130

[11] Ghaleb, F., Daoud, S., Hasna, A., ALJa’am, J. M., El-Seoud, S. A., \& El-Sofany, H. (2006). E-learning model based on semantic web technology. International Journal of Computing \& Information Sciences, 4(2), 63-71.

[12] Guoli, Z., \& Wanjun, L. (2010). The applied research of cloud computing platform architecture in the E-Learning area.

[13] Hendler, J. (2009). Web 3.0 Emerging. Computer, 42(1), 111-113. http://dx.doi.org/10.1109/MC.2009.30

[14] Hwang, G. J., Kuo, F. R., Yin, P. Y., \& Chuang, K. H. (2010). A heuristic algorithm for planning personalized learning paths for context-aware ubiquitous learning. Computers \& Education, 54(2), 404-415. http://dx.doi.org/10.1016/j.compedu.2009.08.024

[15] Kim, P., Ng, C. K., \& Lim, G. (2010). When cloud computing meets with Semantic Web: A new design for e-portfolio systems in the social media era. British Journal of Educational Technology, 41(6), 1018-1028. http://dx.doi.org/10.1111/j.14678535.2010.01055.x

[16] Mahmud, A. H., \& Hassanuzzama, M. (June 2009). The role of Web 2.0 tools in collaborative learning.

[17] Mell, P., \& Grance, T. (2011). The NIST definition of cloud computing (draft). NIST special publication, 800, 145.

[18] Monfort, V., \& Khemaja, M. (2011). Towards Adaptable eLearning Services Invocation: Application to Navigation and Fishing Simulator. Engineering e-Learning Systems, 59. 
[19] Monfort, V., Khemaja, M., Ammari, N., \& Fehli, F. (2010). Using SaaS and Cloud computing For "On Demand" E Learning Services Application to Navigation and Fishing Simulator.

[20] Mrdalj, S. (2011). Would Cloud Computing Revolutionize Teaching Business Intelligence Courses? Navigating Information Challenges, 8, 209.

[21] Torniai, C., Jovanovic, J., Gasevic, D., Bateman, S., \& Hatala, M. (2008). E-learning meets the Social Semantic Web.

[22] WEBER, A. S. (2011). CLOUD COMPUTING IN EDUCATION IN THE MIDDLE EAST AND NORTH AFRICA (MENA) REGION: CAN BARRIERS BE OVERCOME?

\section{AUTHORS}

M. N. is currently an Assistant Professor at Faculty of Computers and Information, Helwan University, Egypt (e-mail: m.nasr@helwan.edu.eg).

S. O. is currently an M.Sc. Candidate at Faculty of Computers and Information, Helwan University, Egypt (email:shimaaouf@yahoo.com).

Submitted on 25 March 2012. Published as resubmitted by the authors on 5 August 2012. 